\title{
El consorcio HIRLAM y el SPC GLAMEPS
}

Gema Morales Martín

Área de Modelización, Agencia Estatal de Meteorología (AEMET)

Carlos Santos Burguete

Centro Nacional de Predicción (CNP), AEMET

Individualmente, somos una gota. Juntos, somos un océano.

\section{Ryunosuke Satoro - ESCRITOR JAPONÉS,}

El desarrollo y mantenimiento de los modelos numéricos de predicción del tiempo es tremendamente complejo, razón por la que han ido surgiendo grandes colaboraciones internacionales para tal fin. Un ejemplo de esta colaboración es el Centro Europeo de Predicción a Plazo Medio (ECMWF, cap. 19 en la página 289), enfocado en modelos globales, ligados al medio plazo. Otro tipo de colaboración se consigue formando consorcios, modalidad en la que los distintos servicios meteorológicos aportan recursos económicos y de personal, se suele trabajar de forma distribuida en los distintos países y se enfoca más bien en modelos regionales o de área limitada, ligados al corto y muy corto plazo. AEMET forma parte del consorcio HIRLAM, consorcio pionero nacido en 1985, que tiene una colaboración muy estrecha con el consorcio ALADIN. Fruto de esta colaboración resulta el Sistema compartido ALADIN-HIRLAM dentro del cual HARMONIE-AROME es una configuración (llamada a veces modelo por sencillez). GLAMEPS, SPC de escala sinóptica, fue el primer SPC fruto de la misma colaboración HIRLAM-ALADIN, con una importante participación española. Presentamos en este capítulo el panorama de HIRLAM, comentando brevemente sobre otros consorcios europeos, así como el SPC sinóptico GLAMEPS, primer SPC paneuropeo.

Palabras clave: High-Resolution Limited-Area Model (HIRLAM), modelo HIRLAM, modelo HARMONIE, consorcio HIRLAM, consorcio ALADIN, consorcio COSMO, consorcio LACE, SPC GLAMEPS, Harmon-EPS. 


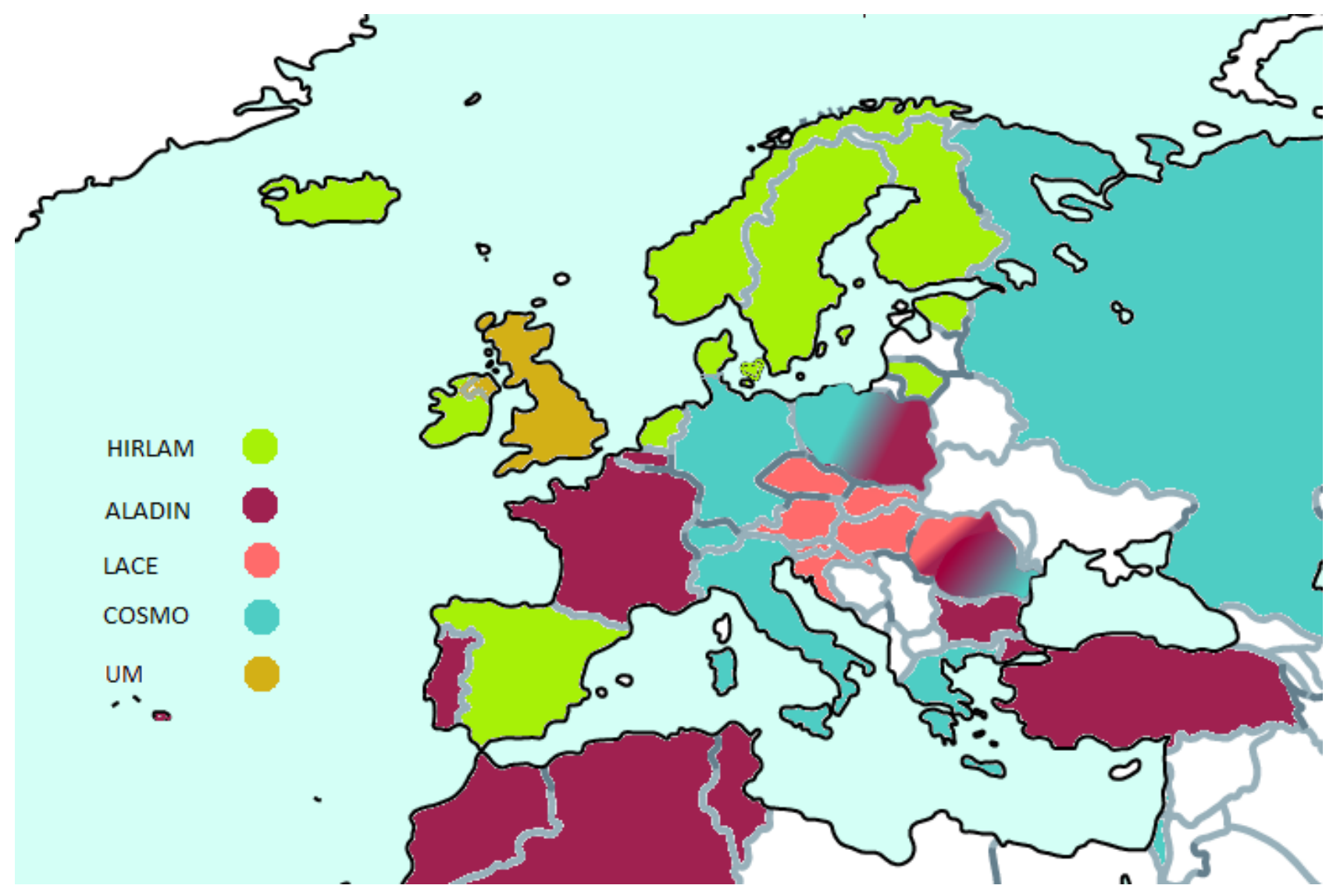

Figura 20.1: Mapa de los consorcios meteorológicos. HIRLAM.

\subsection{El consorcio HIRLAM y otras agrupaciones europeas}

Los primeros modelos utilizados en meteorología fueron los de escala global. Es decir, modelos que representaban la atmósfera a escala planetaria y con una resolución espacio-temporal de los procesos bastante pobre. Un aumento de esta resolución y, por tanto, una mejor descripción de los fenómenos más pequeños, siempre supone un coste computacional mayor. Por ello, la mejora de los modelos globales siempre ha ido emparejada con el avance en la capacidad de cálculo de los ordenadores. Otra manera de incrementar la resolución de un modelo sin necesidad de aumentar el rendimiento de un ordenador sería disminuir el área de integración. Así surgió la idea de utilizar modelos de área limitada, los cuales necesitan de un enfoque diferente y más complejo que un modelo global.

Desde un primer momento, todos los Servicios Meteorológicos Nacionales en Europa tuvieron constancia del esfuerzo que iba a suponer el desarrollo no solo del modelo atmosférico en sí mismo, si no de un sistema de predicción completo para su uso operativo en meteorología. Conscientes de este hecho, los institutos europeos crearon distintas alianzas internacionales entre ellos con el fin de colaborar en el desarrollo de un sistema común de predicción orientado al corto y muy corto plazo.

El consorcio HIRLAM (High-Resolution LimitedArea Model, http://www.hirlam.org/) fue el primero que surgió, en el año 1985, para dotar a sus países miembros de un sistema de predicción numérica de área limitada. Inicialmente estaba constituido por tres países: Finlandia, Noruega y Suecia. En enero de 1990, el servicio meteorológico finlandés fue el primer instituto que puso en marcha de forma operativa el modelo HIRLAM. Aunque habitualmente nos referimos al modelo HIRLAM, no hablamos exclusivamente de las ecuaciones físico-matemáticas que describen a la atmósfera en su conjunto. Sería más correcto hablar del sistema HIRLAM, que además del código con las ecuaciones, está constituido por un complejo sistema de directivas que controlan la ejecución del modelo, herramientas adicionales que preparan las fuentes iniciales que lo alimentan, e. g. observaciones, condiciones iniciales (CCII), etc. y que generan productos posprocesados a partir de las variables de diagnóstico del modelo.

Las actividades del grupo HIRLAM se extendieron 
en el tiempo, organizando las tareas a desarrollar en forma de proyectos que durarían un par de años cada uno. Desde el año 2006, los miembros del consorcio decidieron continuar su colaboración pero con programas de cinco años de duración donde se establecen claramente cuáles son los objetivos a alcanzar al finalizar cada uno de ellos: HIRLAM-A (2006-2010), HIRLAM-B (2010-2015), HIRLAM-C (2016-2020). A lo largo de las últimas tres décadas de existencia del consorcio se fueron uniendo otros países europeos, siendo diez los que actualmente usan de forma operativa el mismo sistema para la predicción a corto plazo: Dinamarca, España, Estonia, Finlandia, Holanda, Islandia, Irlanda, Lituania, Noruega y Suecia.

Existen alianzas similares que aglutinan otros países europeos y a las que a lo largo de su historia poco a poco se les fueron uniendo los distintos servicios meteorológicos. Se ilustran los diferentes consorcios en la Figura 20.1. Hoy en día están constituidos por:

- COSMO (Consortium for Small-scale Modelling, http: //www . cosmo-model.org), compuesto actualmente por Alemania, Grecia, Israel, Italia, Polonia, Rumanía, Rusia y Suiza.

- RC LACE (Regional Centre for Limited Area modelling in Central Europe, http://www.rclace. $\mathrm{eu}$ ), formado por Austria, Croacia, Eslovaquia, Eslovenia, Hungría, República Checa y Rumanía.

Finalmente, el Reino Unido utiliza su propio modelo denominado Unified Model (http://www. metoffice.gov.uk), que corre en distintas configuraciones de forma operativa en su servicio meteorológico.

En 1990, Météo-France, que ya disponía del modelo de escala global ARPEGE, propone a los miembros del consorcio RC LACE colaborar estrechamente para transformar el modelo ARPEGE en uno de área limitada. Un año más tarde, se formaliza esta colaboración conjunta siendo la génesis de lo que se conoce como el consorcio ALADIN (Aire Limitee Adaptation Dynamique Developpement International). A este nuevo grupo se le fueron uniendo más países, y no solo de Europa, estando formado actualmente por dieciséis miembros: Francia, República Checa, Hungría, Rumanía, Austria, Polonia, Bulgaria, Eslovaquia, Eslovenia, Marruecos, Croacia, Bélgica, Portugal, Túnez, Argelia y Turquía.

Pese a las fronteras, el intercambio de conocimientos entre los científicos y desarrolladores de los diferentes consorcios ha sido una constante a lo largo de la historia de la modelización. En los últimos años, los grupos HIRLAM y ALADIN han ido incrementando progresivamente la cooperación entre ellos, formalizando en diciembre de 2005 dicha colaboración. El objetivo del trabajo conjunto sigue siendo el mismo que cada uno de ellos perseguía por separado. Por un lado, mejorar la modelización numérica de los procesos atmosféricos usando sistemas regionales de mayor resolución espacio-temporal, con especial énfasis en la detección y pronóstico de fenómenos severos. Y, por otro lado, desarrollar sistemas eficientes de predicción meteorológica que se puedan usar de manera óptima en los distintos servicios meteorológicos. Fruto de esta acción conjunta surgió el sistema de predicción HARMONIE (HIRLAM-ALADIN Research on Meso-scale Operational NWP in Euromed), que constituye a día de la presente publicación uno de los sistemas operativos más sofisticados a nivel mundial. HARMONIE permite distintas configuraciones de uso y dentro de la comunidad HIRLAM la opción preferente es la conocida como HARMONIE-AROME. Esta es la versión del modelo no hidrostática, que resuelve explícitamente la convección (ya no está parametrizada) y que permite resoluciones del orden de $1 \mathrm{~km}$ en su operatividad. Este modelo actualmente se utiliza de forma determinista, como modelo de predicción de área limitada, además de su uso en su versión de predicción por conjuntos. También existe la opción de correr el modelo de forma climática y, actualmente, existe una línea de desarrollo que incluye la integración de los procesos meteorológicos con la química atmosférica (aerosoles, contaminantes, etc.)

Existe el compromiso de que para el año 2020, ambos consorcios se fusionen en uno solo y doten de un único sistema común de predicción para ser usado en los 26 países. 


\begin{tabular}{ll} 
Tipo & $\begin{array}{l}\text { Superensemble multimodelo con desfase } \\
\text { temporal }\end{array}$ \\
\hline Modelos/Física & $\begin{array}{l}\text { Hirlam STRACO, Hirlam Kain/Fritsch, Alaro } \\
\text { SURFEX, Alaro ISBA }\end{array}$ \\
\hline Tamaño & 54 miembros \\
\hline Subensembles & 4 x $(1+12)$ miembros \\
\hline Pasadas & 4 diarias: 00, 06, 12, 18 UTC \\
\hline Alcances & Hasta T+54 h \\
\hline Resolución horizontal & 7.5 km $(5$ km en v3) \\
\hline Dominio & Pan-europeo \\
\hline CCII y contorno & ECENS (downscaling)
\end{tabular}

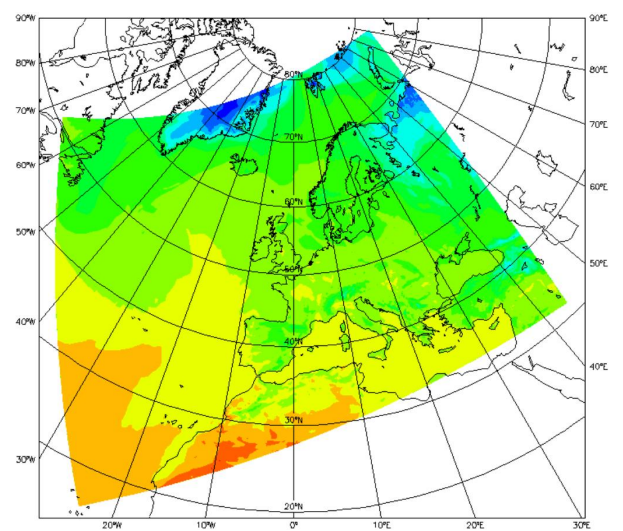

Tabla 20.1: Tabla resumen de características del SPC GLAMEPSv2.

\subsection{GLAMEPS}

El Gran Sistema de Predicción por Conjuntos de Modelos de Área Limitada, Grand Limited Area Model Ensemble Prediction System, GLAMEPS [3], es un proyecto conjunto de los consorcios HIRLAM y ALADIN. Se trata de un Sistema de Predicción por Conjuntos, SPC (cap. 13 en la página 165) paneuropeo por su dominio, siendo el primer SPC de área limitada en cubrir el continente europeo por completo. El proyecto comenzó en 2006 y participan o han participado numerosos países entre los que pueden destacarse Noruega, Dinamarca, España, Suecia, Bélgica, Holanda, Hungría e Irlanda. La versión operativa en 2017 es GLAMEPSv2, aunque está funcionando en paralelo la versión GLAMEPSv3, que se ha desarrollado por el Grupo de Predecibilidad del proyecto HIRLAM. En ese proyecto se viene desarrollando desde hace años una plataforma para poner en funcionamiento SPC de mesoescala en dominios nacionales, plataforma que se denomina Harmon-EPS. La Tabla 20.1 ofrece un resumen básico de las características de GLAMEPS.

\subsubsection{Configuración}

Instalación. GLAMEPS funciona en las plataformas de supercomputación del Centro Europeo de Predicción a Plazo Medio, European Centre for Mediumrange Weather Forecasts, ECMWF (sec. 19.4 en la página 297), en una modalidad de aplicación llamada Time-Critical Facility que se caracteriza, entre otras ventajas, por estar mantenida por personal del centro de proceso de datos del ECMWF. GLAMEPS utiliza el SPC del ECMWF, ECENS (sec. 19.3 en la página 293), tradicionalmente llamado EPS en Agencia Estatal de Meteorología (AEMET) y denominado ENS-IFS en el ECMWF, como condiciones iniciales y de contorno.
Naturaleza. En su etapa de madurez, GLAMEPS es un superensemble multimodelo de 4+48 miembros, con 4 miembros de control más 48 miembros perturbados, incluyendo desfase temporal o lagging (sec. 13.5.2 en la página 174), técnica especial descrita también más adelante, en la mitad de los miembros perturbados. GLAMEPSv2 se lanza en 4 ciclos o pasadas por día, 00, 06, 12 y 18 UTC, donde cada pasada consiste en un superensemble de 4+24 miembros. Para ser precisos, cada pasada consta exactamente de 4 ensembles de 1+6 miembros cada uno. La configuración de miembros está preparada para que 2 pasadas consecutivas constituyan un superensemble de $4+48$ miembros.

Modelos. GLAMEPSv2 se compone de los modelos descritos en la Tabla 20.2. La etiqueta 000 se refiere a miembro de control. La numeración de los miembros coincide con la numeración interna de los 1+50 miembros del ECENS y los LAM de GLAMEPS utilizan los miembros respectivos del ECENS como condiciones iniciales y de contorno. Por tanto, GLAMEPS es un downscaling (sec. 10.1 en la página 130) del ECENS. Todos los miembros tienen su propio ciclo de asimilación en superficie y los miembros de control realizan 3DVAR (sec. 16.2.3 en la página 247) para análisis en altura. Los HIRLAM llevan física estocástica y perturbaciones de las observaciones de superficie. Los miembros de control hacen 3DVAR (ver sec. 16.6 en la página 247).

\begin{tabular}{lcc}
\hline Acrónimo & Modelo & Contribución miembros \\
\hline HirEPS_S & Hirlam STRACO & $000,001-006,007-012$ \\
HirEPS_K & Hirlam K/F & $000,013-018,019-024$ \\
AloEPS_S & Alaro SURFEX & $000,025-030,031-036$ \\
AloEPS_I & Alaro ISBA & $000,037-042,043-048$ \\
\hline
\end{tabular}

Tabla 20.2: Modelos integrantes de GLAMEPSv2. 


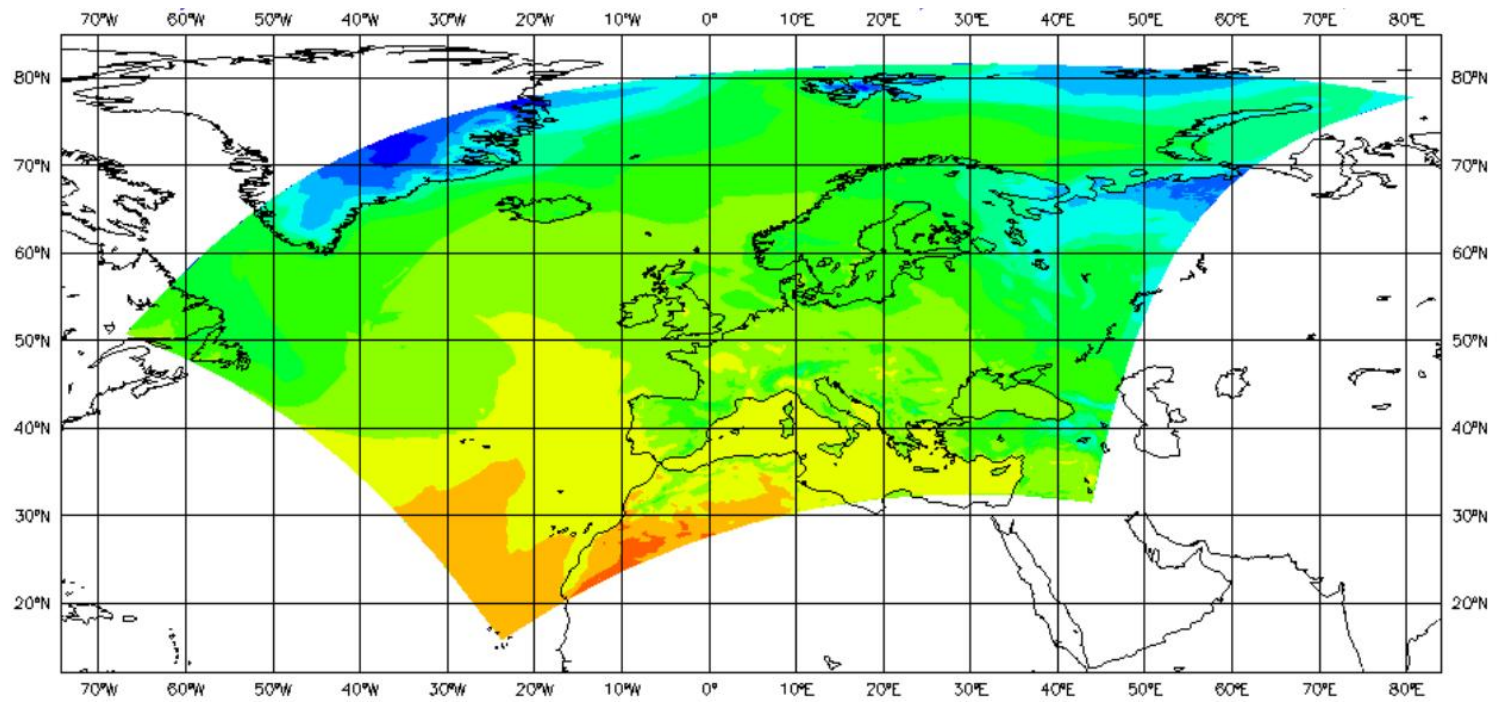

Figura 20.2: Dominio Pan-europeo de GLAMEPSv2 en proyección cilíndrica.

Dominio. El dominio de GLAMEPSv2 es paneuropeo, abarcando en diagonal holgadamente desde las islas Canarias (gracias a nuestra participación en el proyecto, de otro modo se habría desconsiderado) hasta las islas Svalbard y desde Terranova hasta Oriente Medio. Queda ilustrado en dos proyecciones distintas en la Tabla 20.1 en la página anterior (figura interior) y en la Figura 20.2.

Malla. En el caso de los modelos HIRLAM se trata de una malla rotada latitud-longitud (latlon rotada) para minimizar el impacto de la variabilidad del tamaño de celda con la latitud. El polo sur se sitúa en $(-46,30)$. En el caso de los ALARO se trata de una rejilla lambert conforme con $7.5 \mathrm{~km}$ de resolución natural. El número de puntos en la malla es 388 × $242=93896$

Resolución. Horizontal: $0,075^{\circ} \times 0,075^{\circ}$ en la rejilla natural de HIRLAM y $7.5 \mathrm{~km}$ en la rejilla natural de ALARO. Vertical: 60 niveles híbridos.

\begin{tabular}{cllll}
\hline Pasada & \multicolumn{4}{c}{ Miembros perturbados } \\
\hline & HirEPS_S & HirEPS_K & AloEPS_S & AloEPS_I \\
00 & $001-006$ & $013-018$ & $025-030$ & $037-042$ \\
06 & $007-012$ & $019-024$ & $031-036$ & $043-048$ \\
\hline
\end{tabular}

Tabla 20.3: Distribución de miembros perturbados para las distintas pasadas de un día en GLAMEPSV2.

Construcción de las pasadas (Cycling). GLAMEPSv2 no ejecuta todos los miembros en cada pasa- da, como se hacía en GLAMEPSv1. En GLAMEPSv2 los miembros perturbados están separados en dos grupos, que se lanzan alternativamente en las pasadas 00, 12 ó 06, 18 UTC. La Tabla 20.3 muestra la distribución de miembros perturbados para las distintas pasadas de un día. Los miembros (no perturbados) de control para cada uno de los 4 modelos, miembros identificados con la etiqueta 000 , se lanzan en cada pasada.

Esta configuración conduce a un superensemble completo cuando se combinan los miembros perturbados de dos pasadas consecutivas, y se añaden los miembros de control de la pasada más reciente, según ilustra la Figura 20.3. De este modo, GLAMEPS es capaz de proporcionar predicciones (productos) probabilistas cada 6 horas, a la vez que optimiza el uso de los recursos computacionales.

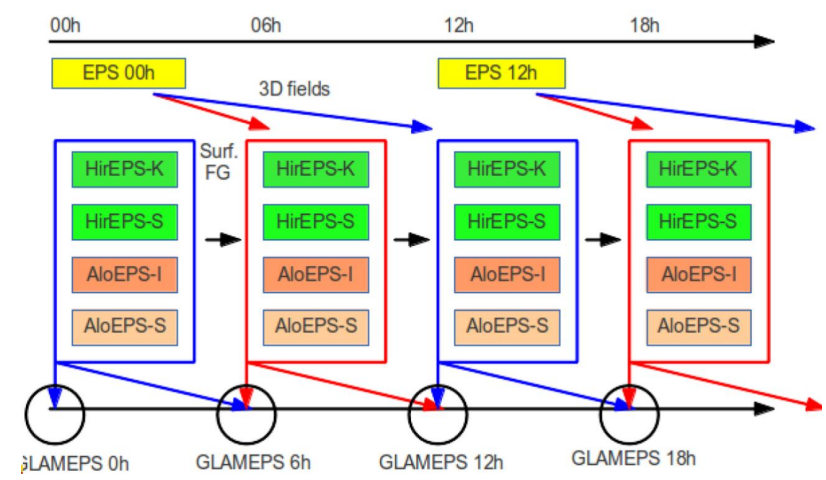

Figura 20.3: Construcción de las diferentes pasadas. Imagen cedida por INGER-LISE FROGNER, 2014. 


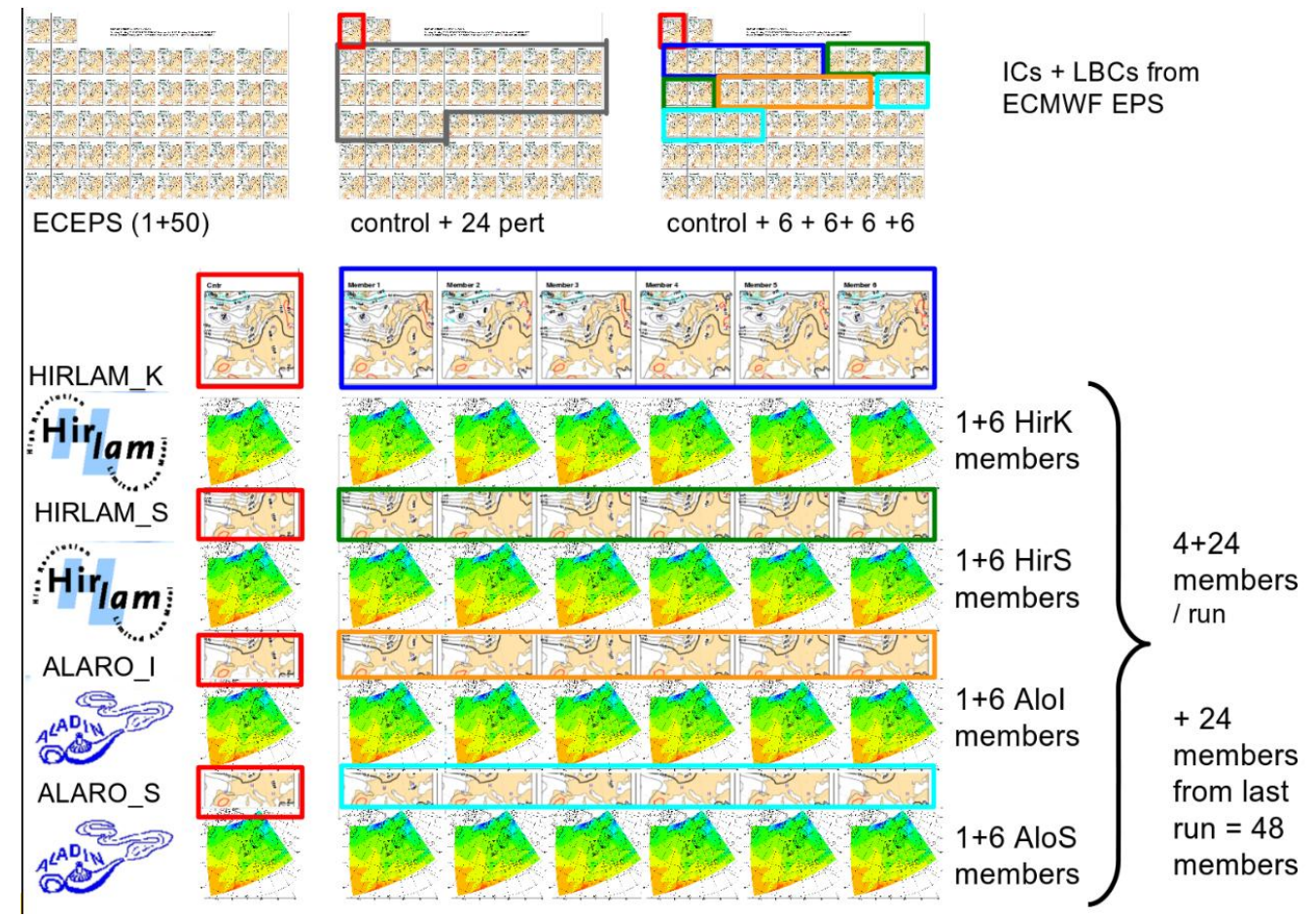

Figura 20.4: Esquema completo de GLAMEPSv2, con la construcción de las diferentes pasadas a partir de distintos miembros de ECENS (ver texto).

GLAMEPS hace downscaling (sec. 10.1 en la página 130) de ECENS: toma condiciones iniciales y de contorno de los miembros de ECENS, de modo que en diferentes pasadas toma subconjuntos distintos de esos miembros. Este esquema se ilustra en la Figura 20.4. En la parte superior podemos ver los típicos sellos (sec. 27.2.1 en la página 402) del ECENS, a su derecha la selección para una pasada de GLAMEPS y, a su derecha, la subdivisión dentro de esa selección, con colores correspondientes a la tabla coloreada inferior. En dicha tabla, cada fila de las 4 muestra un subensemble basado en un modelo (dos HIRLAM, dos ALARO). Cada subensemble consta de un miembro de control, downscaling del miembro de control de ECENS, en rojo, más 6 miembros perturbados, downscaling de 6 miembros perturbados de ECENS, en distintos colores, uno para cada subensemble. Después, tal como se describía anteriormente, se añaden los 6 miembros perturbados de cada subensemble de la pasada anterior (time-lagging), para formar los cuatro Subensembles de 1+12 miembros cada uno. En total GLAMEPS consta de $4 \times(1+12)=54$ miembros, equiparable a ECENS que tiene 51.

Flexibilidad. Dado que los datos de los miembros están disponibles separadamente, cada usuario puede elegir la combinación o conjunto que más le convenga y aplicar su propio posproceso.

\subsubsection{Verificación}

En los diversos ejercicios de verificación realizados, GLAMEPS vino obteniendo mejor rendimiento que AEMET-SREPS y que ECENS. Así mismo, las versiones calibradas convenientemente obtuvieron mejores resultados que las versiones crudas. Su naturaleza mixta con multimodelo y perturbaciones, el hecho de ser un downscaling de ECENS y, por supuesto, su resolución superior de $7.5 \mathrm{~km}$ explican la mejor calidad en las salidas.

En la Figura 20.5 en la página siguiente se muestra un ejemplo de ejercicio de verificación. En la gráfica izquierda se muestra la relación dispersión-error (sec. 15.8.2 en la página 223), GLAMEPS en azul y ECENS en verde, mostrando GLAMEPS una relación mejor, más cercana a la diagonal. En la gráfica derecha se muestra el BSS (sec. 15.7 en la página 224) de las probabilidades de viento por encima de $10 \mathrm{~m} / \mathrm{s}$, esta vez GLAMEPS en verde, AEMET-SREPS en rojo y ECENS en azul; para todos los alcances de predicción AEMET-SREPS mejora el rendimiento de ECENS y GLAMEPS mejora a su vez el de AEMETSREPS, consistentemente, como decíamos previamente, con sus resoluciones horizontales de $50 \mathrm{~km}, 25 \mathrm{~km}$ y $11 \mathrm{~km}$ en la época del ejercicio. 

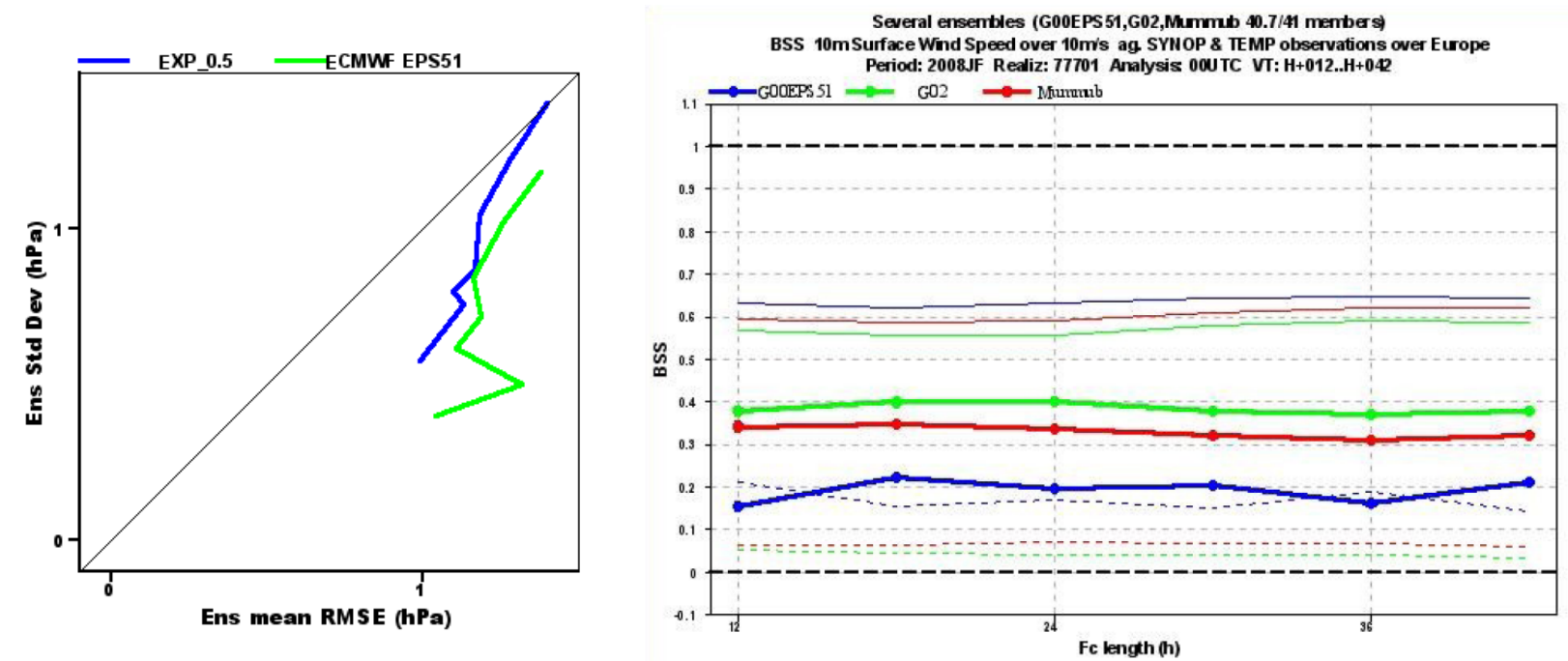

Figura 20.5: Verificación de GLAMEPS (ver texto).

\subsubsection{Calibración}

Viento. El posproceso estadístico pasó de realizarse con BMA (sec. 14.1.2.0.1 en la página 195 y 21.6 en la página 319) a realizarse utilizando la regresión logística extendida, extended logistic regresion, ELR (sec. 14.1.2.0.7 en la página 198). Se utilizan como predictores el promedio del SPC, la latitud, la longitud y la altitud de la estación. Para ejercicios de periodos de entrenamiento del orden de un mes y verificación similar, con estaciones europeas para el alcance predictivo de $\mathrm{T}+18 \mathrm{~h}$ las mejoras son sustanciales, tanto en fiabilidad como en resolución (sec. 15.8 en la página 224). Pueden mejorarse los resultados para umbrales altos entrenando en áreas pequeñas, resultado mostrado en la Figura 20.6. Entrenando en el rectángulo que comprende parte de Alemania, los resultados mejoran. La gráfica muestra el BSS (sec. 15.8 en la página 224) con respecto al umbral en $\mathrm{m} / \mathrm{s}$. Con área de entrenamiento de toda Europa, 100000 casos, en rojo, puede verse la degradación progresiva y, a partir de $10 \mathrm{~m} / \mathrm{s}$ el rendimiento muy bajo. Reduciendo el área a una de 25000 casos, en negro discontinuo, la mejora es notable. Añadiendo, además, técnicas spline, en negro continuo, se mejora notablemente el rendimiento también para umbrales bajos.

Temperatura. Para la calibración específica en temperatura a $2 \mathrm{~m}$ se aprovechó el ejercicio de predicción de los juegos olímpicos de Sochi, Figura 20.7 en la página siguiente. Se calibraba con datos de 30 estaciones y se actualizaba la predicción con mucha frecuencia, lo que mejoraba ostensiblemente el rendimiento, en rojo, con respecto al original, en negro.
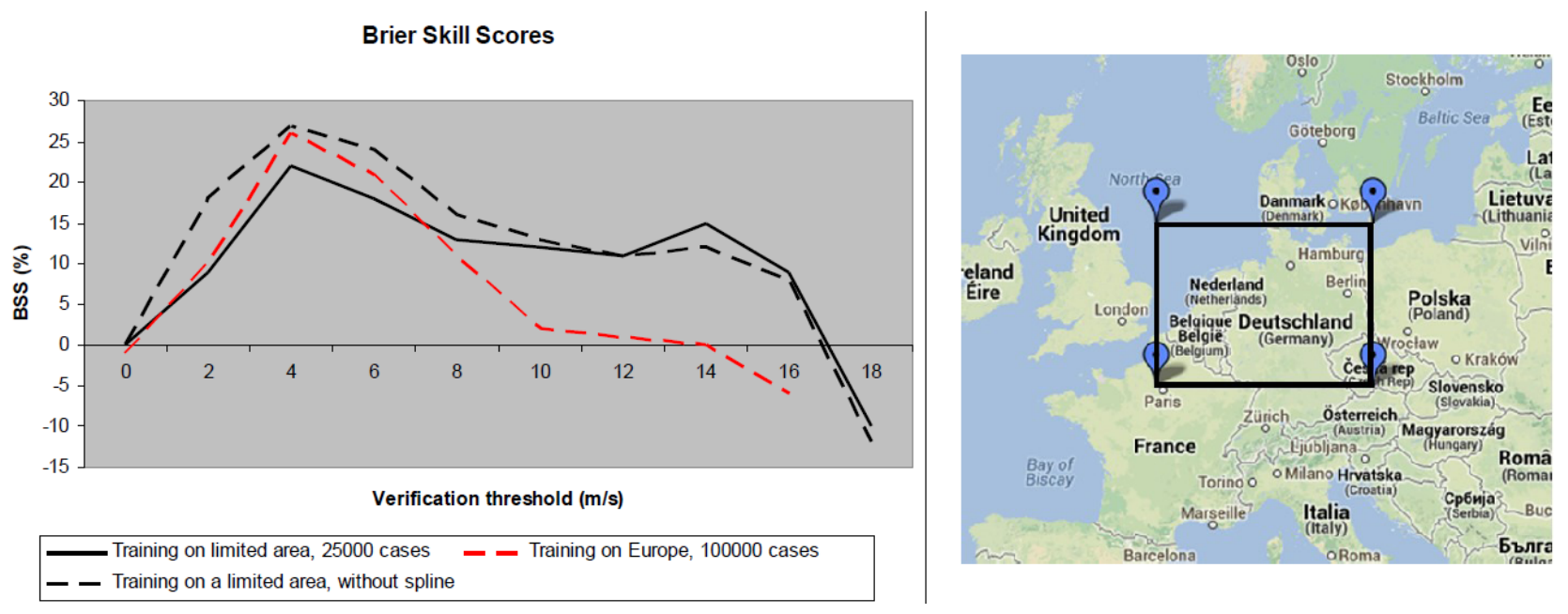

Figura 20.6: Calibración del viento a $10 \mathrm{~m}$. Realizando el entrenamiento en áreas relativamente pequeñas, los resultados para umbrales altos pueden mejorar (ver texto). FROGNER, 2014. 

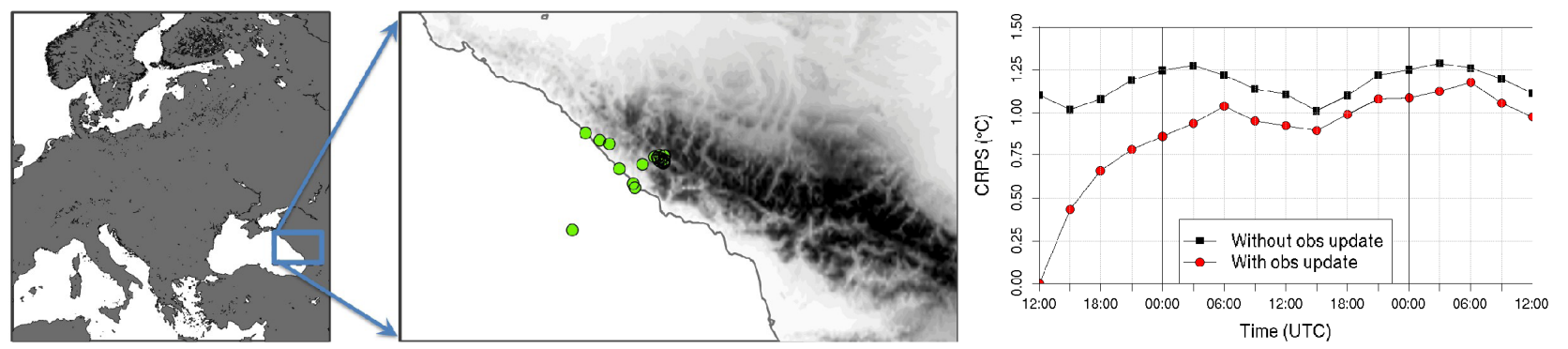

Figura 20.7: Calibración de la temperatura a 2 m. FROGNER, 2014.

\subsubsection{Ejemplos de predicciones}

Olas de calor. En la emisión de avisos de temperaturas máximas en la temporada estival, la resolución horizontal de los SPC juega un papel decisivo. La temperatura a $2 \mathrm{~m}$ es un parámetro muy orográfico, es decir, está muy influido por la orografía del modelo.
En la Figura 20.8 podemos observar probabilidades de exceder umbrales de temperatura a $2 \mathrm{~m}$, tanto de ECENS, a $16 \mathrm{~km}$, como de GLAMEPS, a $7.5 \mathrm{~km}$. La capacidad de GLAMEPS de resolver contornos geográficos con algo más del doble de resolución se hace patente en las señales de probabilidad de excedencia de umbrales.

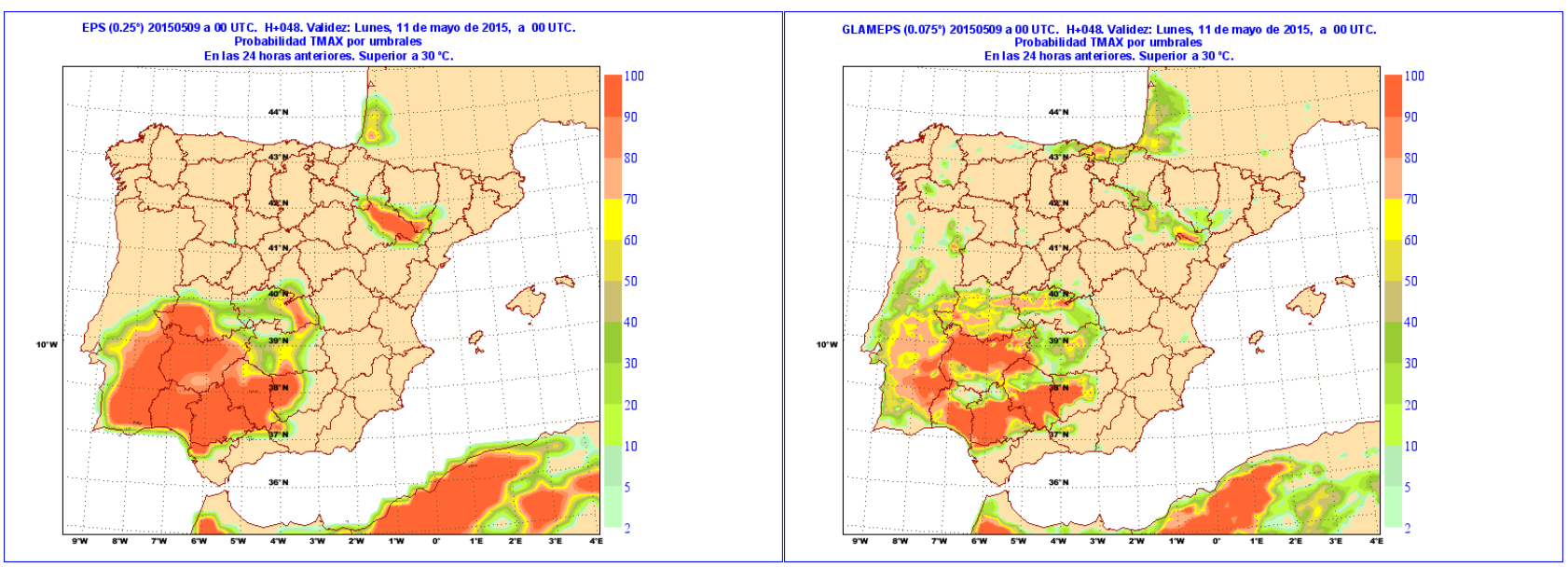

Figura 20.8: Probabilidad de temperaturas por encima de $30^{\circ} \mathrm{C}$ como herramienta de decisión para emisión de avisos. A la izquierda ECENS con resolución horizontal de $16 \mathrm{~km}$. A la derecha GLAMEPS con $7.5 \mathrm{~km}$. La capacidad de GLAMEPS para afinar por pequeñas regiones es notable (ver texto).

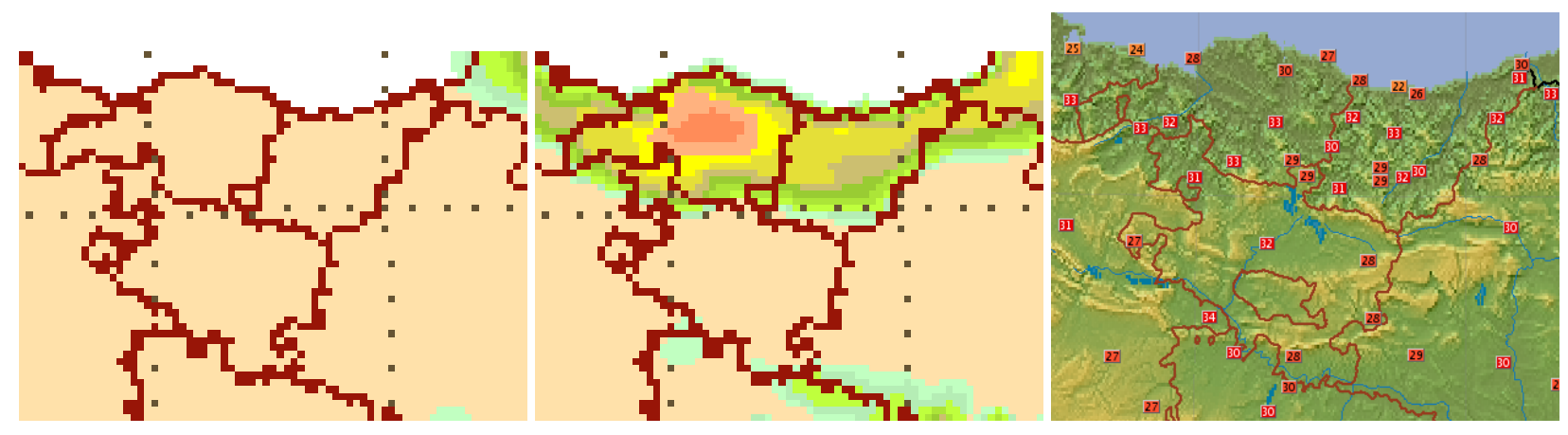

Figura 20.9: Ampliación del País Vasco correspondiente a los mapas de la Figura 20.8, a la izquierda ECENS, en el centro GLAMEPS. Se acompaña, a la derecha, el registro de observaciones que corrobora el rendimiento de GLAMEPS (ver texto). 


\subsubsection{Plataforma Harmon-EPS}

GLAMEPS es, en la actualidad (2018), el último SPC de área limitada capaz de cubrir Europa entera con resoluciones razonablemente altas. Por debajo de los $5 \mathrm{~km}$, al traspasar claramente la frontera de la mesoescala gamma, se requiere que los modelos sean no hidrostáticos (sec. 6.3.2 en la página 78) y el coste computacional se desborda y se requiere cambiar el planteamiento completamente.

La plataforma Harmon-EPS en ensembles es una concepción similar a la plataforma HARMONIE para modelos deterministas. Estas plataformas proporcionan los recursos necesarios para que, con las particularizaciones convenientes, cada país pueda lanzar un modelo o un SPC de altísima resolución en la mesoescala en un dominio reducido acorde con sus necesidades. Así mismo, los esfuerzos comunes para desarrollar estas plataformas revierten positivamente en los participantes. AEMET ha venido participando activamente tanto en GLAMEPS como en Harmon-EPS, en convergencia con los intereses y planes delineados para el AEMET- $\gamma$ SREPS (cap. 22 en la página 333). Se detallan a continuación los aspectos técnicos en que se ha focalizado la plataforma Harmon-EPS.

- Transición a la mesoescala gamma. SPC que permitan resolver la convección explícitamente (convection-permitting).

- Áreas subeuropeas, tendencia hacia áreas pequeñas. Resolución de $2.5 \mathrm{~km}$.

- Dos pasadas diarias, 00 y 12 UTC. Alcance predictivo hasta $\mathrm{T}+36 \mathrm{~h}$.

- Asimilación de datos completa y ciclos de 3 h para el miembro de control. Asimilación de superficie para todos los miembros.

- En elcaso particular de AEMET- $\gamma$ SREPS (cap. 22 en la página 333), continuar con el paradigma multimodelo: 10 miembros AROME + 10 miembros ALARO $=20$ miembros

- Perturbaciones en las CCII: EDA como referencia y, alternativamente, LETKF (sec. 16.2.2 en la página 246), 4DVAR y downscaling del ECENS.

- Perturbaciones en el modelo: SPPT (sec. 17.4.4 en la página 269) y multifísica.

- Parametrizaciones estocásticas para la convección profunda organizada usando el algoritmo de autómata celular $[1,2]$.

- Perturbaciones en las condiciones de contorno: comparativas entre ECENS T639 vs ECENS T1279.

\subsubsection{Anexo: GLAMEPS en cifras}

Volumen de operación diaria. Hay ficheros correspondientes a 4 subensembles, en total 54 miembros, 2 pasadas (00 y 12 UTC), con 18 alcances predictivos (de 3 h a 54 h cada 3 h), lo que hace 54 x 2 x $18=1944$. Cada fichero contiene datos de, al menos 18 parámetros, un total de 1944 x $18=34992$ bloques de parámetro dentro de 1944 ficheros. Considerando que un fichero tiene un peso medio de $21 \mathrm{Mb}$, tendremos 1944 x $21 \mathrm{Mb}=40 \_824 \_\mathrm{Mb} \sim 40 \mathrm{~Gb}$ diarios.

Volumen para verificación mínima. Para verificación se selecciona un subconjunto mínimo de parámetros por razones de austeridad de espacio en disco, de modo que se almacenan únicamente 8 parámetros de los 18 operativos. Tendremos entonces 1944 x $8=15552$ bloques de parámetro dentro de 1944 ficheros. Considerando que un fichero tiene un peso medio de $9 \mathrm{Mb}$, tendremos $17 \mathrm{~Gb}$ diarios, $520 \mathrm{~Gb}$ al mes y $6 \mathrm{~Tb}$ al año.

Información gráfica. La cobertura gráfica de un ejercicio completo de verificación es un auténtico problema dimensional. Solamente para la parte determinista se debe pintar sesgo y error cuadrático medio de todos los miembros y promedio del SPC. En la verificación propiamente probabilista está la parte de flujo a gran escala por un lado, típicamente para z500, t500, t850 y mslp, dispersión-error e histogramas de rango y, por otro lado, las variables de tiempo sensible temperatura, viento y precipitación, con desdoblamiento por umbrales (e. g. pcp $>=0.3,1,5,10 \ldots$ ), diagramas de fiabilidad, agudeza, ROC, RV, BS y BSS y sus descomposiciones. El resultado aproximado es un mínimo de 4500 gráficas, eligiendo lo más sencillo. En la práctica nadie puede enseñar, en un artículo, libro o conferencia 4500 gráficas con información de rendimiento y otras medidas. Se suelen seleccionar algunas que sean representativas de algún aspecto a resaltar.

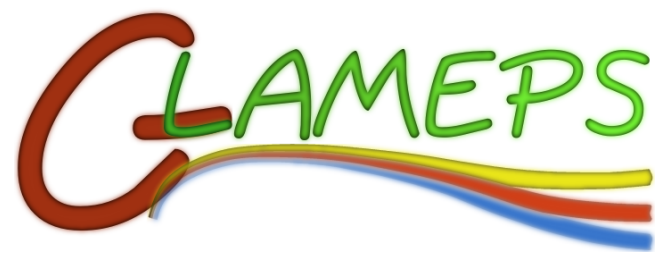

Figura 20.10: Logo de GLAMEPS, idea original de KAI SATLER $(D M I)$. 


\begin{tabular}{|c|c|c|c|c|c|}
\hline modelo & \multicolumn{3}{|c|}{ HIRLAM } & \multicolumn{2}{|c|}{ HARMONIE-AROME } \\
\hline dominio & ONR & HNR & $\mathrm{CNN}$ & Iberia & Canarias \\
\hline malla & $0.17\left[^{\circ}\right]$ & $0.55\left[^{\circ}\right]$ & $0.055\left[^{\circ}\right]$ & $2.5[\mathrm{~km}]$ & $2.5[\mathrm{~km}]$ \\
\hline puntos de malla & $582 \times 424$ & $606 \times 430$ & $606 \times 430$ & $1152 \times 864$ & $576 \times 480$ \\
\hline niveles verticales & \multicolumn{3}{|c|}{40} & \multicolumn{2}{|c|}{65} \\
\hline pasadas & \multicolumn{3}{|c|}{$00 / 06 / 12 / 18$} & \multicolumn{2}{|c|}{ 00/03/06/09/12/15/18/21 } \\
\hline alcances & $+72 \mathrm{~h}$ & $+36 \mathrm{~h}$ & $+36 \mathrm{~h}$ & $+48 \mathrm{~h}$ & $+12 \mathrm{~h}$ \\
\hline asimilación & \multicolumn{3}{|c|}{ 3D-VAR } & \multicolumn{2}{|c|}{ 3D-VAR } \\
\hline $\begin{array}{l}\text { condiciones iniciales } \\
\text { y de contorno }\end{array}$ & \multicolumn{3}{|c|}{ ECMWF/IFS } & \multicolumn{2}{|c|}{ ECMWF/IFS } \\
\hline actualización cccc & \multicolumn{3}{|c|}{$3 \mathrm{~h}$} & \multicolumn{2}{|c|}{$1 \mathrm{~h}$} \\
\hline \multirow{2}{*}{ supercomputador } & \multicolumn{3}{|c|}{ Cray X1E (2004-2016) } & \multirow{2}{*}{\multicolumn{2}{|c|}{$\begin{array}{c}\text { Bullx } \\
\text { Ivy Bridge }\end{array}$}} \\
\hline & \multicolumn{3}{|c|}{ Bullx Ivy Bridge } & & \\
\hline
\end{tabular}

Tabla 20.4: Características principales de los modelos HIRLAM y HARMONIE-AROME en AEMET en 2017.
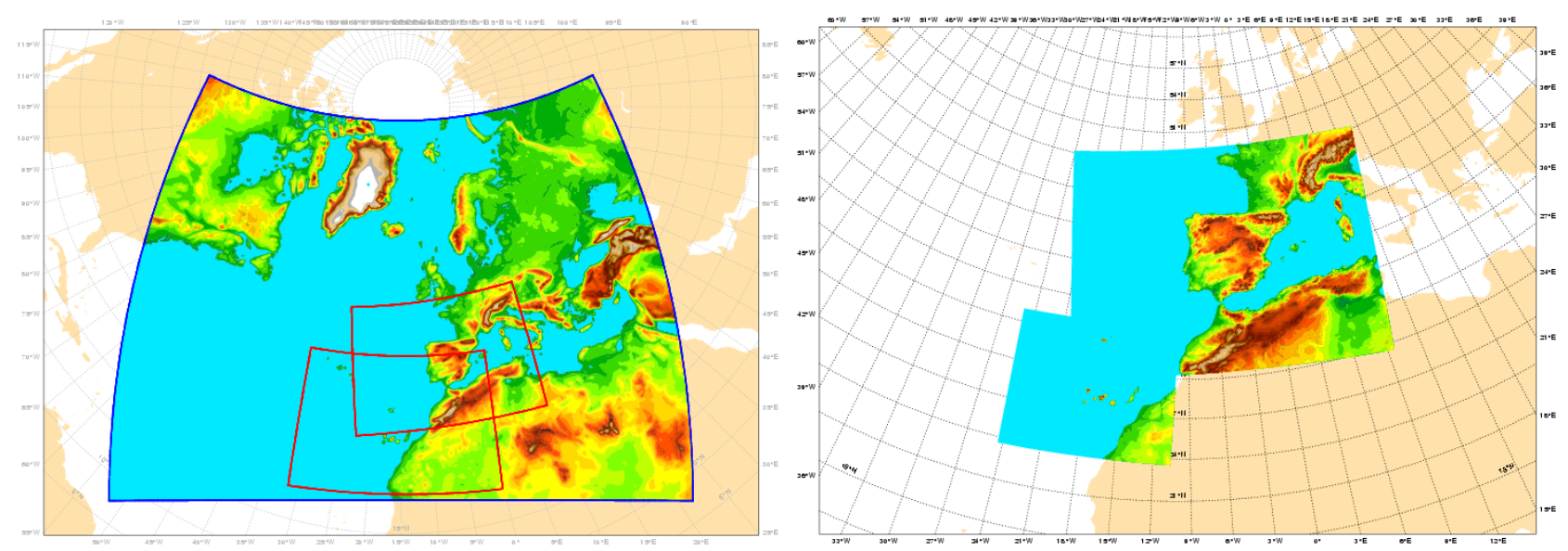

Figura 20.11: Dominios de integración de los modelos integrados en AEMET: HIRLAM la izquierda, HARMONIEAROME a la derecha. (ampliación en Figura 20.12 en la página siguiente). AEMET.

\subsection{Anexo: El modelo HIRLAM en España}

La Tabla 20.4 muestra las principales características de los distintos dominIos de integración de HIRLAM. En las Figuras20.11 y 20.12 en la página siguiente, parte superior, podemos ver los diferentes dominios de integración del modelo HIRLAM en AEMET.

\subsection{Anexo: El modelo HARMONIE- AROME en España}

La Tabla 20.4 muestra las principales características de los diferentes dominos de integración de HARMONIE-AROME. En la Figura 20.12 en la página siguiente, parte inferior, podemos ver los distintos dominios de integración del modelo HARMONIEAROME en AEMET. 

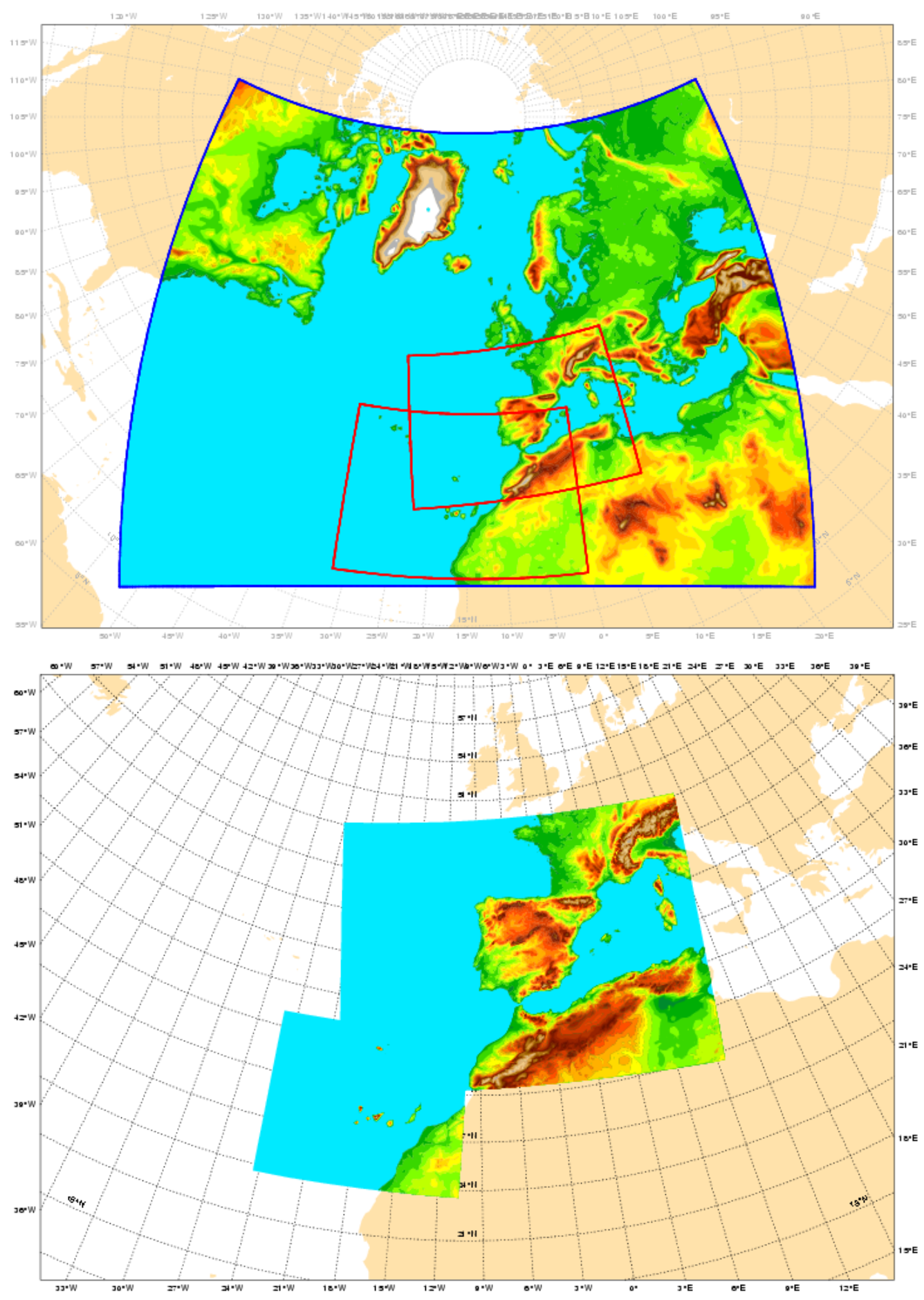

Figura 20.12: Dominios de integración de los distintos modelos en AEMET. Arriba: modelo HIRLAM, dominio ONR en contorno azul, cubriendo buena parte del océano Atlántico Norte, Europa y norte de África, dominio HNR en contorno rojo centrado en la península ibérica y dominio CNN en contorno rojo centrado en las islas Canarias. Abajo: modelo HARMONIE-AROME, un dominio centrado en la península ibérica y el otro centrado en las islas Canarias. AEMET. 


\subsection{Referencias}

[1] Bengtsson, Lisa y col. "A stochastic parametrization for deep convection using cellular automata”. En: Quarterly Journal of the Royal Meteorological Society 139.675 (2013), páginas 1533-1543. DOI: 10.1002/ qj 2108 (citado en página 309).
[2] Gutowitz, Howard. Cellular automata: theory and experiment. MIT press, 1991 (citado en página 309).

[3] IVERSEN, Trond y col. "Evaluation of 'GLAMEPS'-a proposed multimodel EPS for short range forecasting". En: Tellus, Series A: Dynamic Meteorology and Oceanography 63.3 (mayo de 2011), páginas 513-530. ISSN: 02806495. DOI: 10 . 1111/j.1600-0870.2010.00507.x (citado en página 304). 
